\title{
Fuzzy observer for state estimation of the METANeT traffic model
}

\author{
Z. Hidayat, Zs. Lendek, R. Babuška, B. De Schutter
}

\begin{abstract}
Traffic control has proven an effective measure to reduce traffic congestion on freeways. In order to determine appropriate control actions, it is necessary to have information on the current state of the traffic. However, not all traffic states can be measured (such as the traffic density) and so state estimation must be applied in order to obtain state information from the available measurements. Linear state estimation methods are not directly applicable, as traffic models are in general nonlinear. In this paper we propose a nonlinear approach to state estimation that is based on a TakagiSugeno (TS) fuzzy model representation of the METANET traffic model. By representing the METANET traffic model as a TS fuzzy system, a structured observer design procedure can be applied, whereby the convergence of the observer is guaranteed. Simulation results are presented to illustrate the quality of the estimate.
\end{abstract}

\section{INTRODUCTION}

Significant amounts of time and fuel are wasted due to traffic jams, which also contribute to the deterioration of the air quality and environment. Hence, effective traffic control on freeways is necessary to reduce congestion. In this context, traffic control is an important component of the traffic management system that aims at making a better use of the available infrastructure.

Appropriate traffic control actions must be based on the actual traffic state, which, however, is not always available at any point in the traffic network. Not all relevant state variables can be measured due to technical limitations, such as the sparse arrangement of sensors or the occurrence of sensor failures. Moreover, the available measurements are corrupted by noise. For these reasons, traffic state estimation is a very relevant topic with regard to effective traffic control.

Designing a state estimator requires a traffic model. Traffic models are generally classified into microscopic, mesoscopic, and macroscopic models [1]. In the case of on-line modelbased traffic control, it is common to use the macroscopic traffic flow model, see, e.g., [2]-[4]. Such a model is typically nonlinear and captures the average traffic behavior through aggregated variables at different locations in the network [1]. The variables used in macroscopic models include the flow, density, and speed. The model used in this paper is the well-known METANET model [5].

Among the methods applied traffic state estimation are the extended Kalman filter (KF) [6], the unscented Kalman filter [7] and the particle filter [8]. In [9] an adaptive approach to the extended Kalman filter was used. In [10] different filter configurations are compared for the case of traffic flow estimation and parameter estimation.

The authors are with Delft Center for Systems and Control, Delft University of Technology, Mekelweg 2, 2628 CD Delft, The Netherlands Email: z.hidayat@tudelft.nl
A common limitation of the above approaches is the lack of convergence guarantees. Although a well-tuned extended $\mathrm{KF}$, unscented $\mathrm{KF}$, or particle filter can perform well in simulations, there is no guarantee that they will perform equally well in real-life situations. In this paper we propose an alternative approach that is based on transforming the Metanet traffic model into a Takagi-Sugeno (TS) fuzzy model representation and consequently applying a systematic observer design method with stability guarantees. The TS model [11] is a general function approximator that can exactly represent or approximate to an arbitrary degree of accuracy a large class of nonlinear systems. The TS model consists of fuzzy if-then rules. The rule antecedents partition a given subspace of the model variables into fuzzy regions. The consequents of the rules are linear or affine models that are valid locally in the corresponding fuzzy region.

In the literature, there are several approaches to design TS fuzzy observers for continuous-time systems [12] and for discrete-time systems [13]. The design of TS fuzzy observers is formulated as a feasibility problem of Linear Matrix Inequalities (LMI), which can be solved by convex optimization algorithms.

In this paper, we develop a TS fuzzy observer for the METANET traffic flow model. The design starts by transforming the Metanet model into the TS fuzzy model. Then a discrete-time fuzzy observer is designed by applying stability and robustness conditions. This method is the discrete-time counterpart of the approach proposed in [14]. While in [14] the METANET model was first transformed into a continuoustime model, here we design the TS observer directly in the discrete-time setting. This is a much more realistic approach, as the MEtAnet model is in its essence a discrete-time model validated for sampling times that are typically in the order of $10 \mathrm{~s}$. In addition, the measurements are available at discrete time instants as well. It is also important to note that discrete-time design for the TS observer is substantially different from its continuous-time counterpart.

This paper is organized as follows. After the introduction in Section I, the TS fuzzy model and the METANET model are briefly presented in Section II. The TS representation of the Metanet model is derived in Section III. The observer design for a TS fuzzy system is addressed in Section IV. Section V illustrates the proposed approach through a simple case and Section VI concludes the paper.

\section{PRELIMINARIES}

In this section, we briefly review the fuzzy TS model and the Metanet model. 


\section{A. TS fuzzy models}

The Takagi-Sugeno fuzzy model [11] is a mathematical model that can be used to represent nonlinear systems by fuzzy if-then rules with local linear or affine consequents.

Model rule $i$ :

If

$$
z_{1}(k) \text { is } M_{i 1} \text { and } \cdots \text { and } z_{p}(k) \text { is } M_{i p}
$$

Then

$$
\left\{\begin{array}{l}
x(k+1)=A_{i} x(k)+B_{i} u(k)+a_{i} \\
y(k)=C_{i} x(k)
\end{array} \quad i=1, \ldots, r\right.
$$

where $r$ denotes the number of rules, $M_{i j}$ are fuzzy sets, and $A_{i} \in \mathbb{R}^{n \times n}, B_{i} \in \mathbb{R}^{n \times m}, C_{i} \in \mathbb{R}^{q \times n}$, and $a_{i} \in \mathbb{R}^{n}$ are parameters of the local models.

The state and the output of the TS fuzzy system are computed as follows:

$$
\begin{aligned}
x(k+1) & =\sum_{i=1}^{r} h_{i}(z)\left\{A_{i} x(k)+B_{i} u(k)+a_{i}\right\} \\
y(k) & =\sum_{i=1}^{r} h_{i}(z) C_{i} x(k)
\end{aligned}
$$

with

$$
h_{i}(z)=\frac{w_{i}(z)}{\sum_{i=1}^{r} w_{i}(z)}
$$

and

$$
w_{i}(z)=\prod_{j=1}^{p} \mu_{i j}\left(z_{j}(k)\right)
$$

where $\mu_{i j}\left(z_{j}(k)\right)$ denotes the membership function of $M_{i j}$.

In Section III we show how the parameters of the TS fuzzy model can be computed such that it exactly represents a given nonlinear system.

\section{B. The Metanet traffic model}

In this section, we present the METANET traffic flow model developed in [5]. The MEtanet model is one of the existing macroscopic traffic flow models. Macroscopic models express the average behavior of vehicles at a specific location and time instant. Three state variables reflect the behavior of the traffic, namely [5]

- traffic density $\rho$ : the number of vehicles per length unit.

- space-mean speed $v$ : the instantaneous average speed of vehicles in a length increment.

- traffic volume or flow $q$ : the number of vehicles passing/leaving a specific location in each time step.

The Metanet model represents a freeway network as a directed graph whose links are associated with stretches in the freeway network. Each link in the graph corresponds to a stretch that has uniform characteristics. A node is placed in the graph when there is change in the geometry, such as an on-ramp or a split.

The Metanet model is discrete in time and space. In the model, the $m$-th link of a freeway is divided into $N$ segments of length $L_{m}$. For each link $m$ and segment $i$, the state variables of the traffic as described above are expressed as the average density $\rho_{m, i}(k)$, the space-mean speed $v_{m, i}(k)$, and flow $q_{m, i}(k)$. The definitions of the variables that are used in the Metanet model are shown in Table I while the parameters and their typical values (as used in this paper) are given in Table II. The values of the parameters have been adapted from [15].

In segment $i$ of link $m$, the flow at time step $k$ is determined by the speed, the density, and the number of the lanes for the same time step $k$ :

$$
q_{m, i}(k)=\rho_{m, i}(k) \cdot v_{m, i}(k) \cdot \lambda_{m}
$$

where $\lambda_{m}$ is the number of lanes in the corresponding segment. At time step $k+1$, the density of segment $i$ is influenced by the density at time step $k$, the number of vehicles entering from segment $i-1$ (inflow), and the number of vehicles leaving the segment $i$ (outflow). This relationship can be expressed as

$$
\rho_{m, i}(k+1)=\rho_{m, i}(k)+\frac{T}{L_{m} \lambda_{m}}\left(q_{m, i-1}(k)-q_{m, i}(k)\right)
$$

where $T$ is the sampling time, which typically has a value of $10 \mathrm{~s}$. The space-mean speed on segment $i$ at time step $k+1$ is influenced by three terms, expressing relaxation, convection, and anticipation. The relaxation term expresses the speed change in order to achieve a desired equilibrium speed $V\left(\rho_{m, i}(k)\right)$ corresponding to the density $\rho_{m, i}(k)$. This term is proportional to the difference between the current spacemean speed and $V\left(\rho_{m, i}(k)\right)$. The convection term expresses the speed difference between the segment $i$ and the upstream segment $i-1$. The anticipation term is the speed change due to the density change when moving from the upstream segment $i-1$ to the downstream segment $i$. Using these terms, the space-mean speed at time step $k+1$ can be written as

$$
\begin{aligned}
v_{m, i}(k+1) & =v_{m, i}(k)+\frac{T}{\tau}\left[V\left(\rho_{m, i}(k)\right)-v_{m, i}(k)\right] \\
& +\frac{T}{L_{m}} v_{m, i}(k)\left(v_{m, i-1}(k)-v_{m, i}(k)\right) \\
& -\frac{v \cdot T}{\tau \cdot L_{m}} \frac{\rho_{m, i+1}(k)-\rho_{m, i}(k)}{\rho_{m, i}(k)+\kappa}
\end{aligned}
$$

where

$$
V\left(\rho_{m, i}(k)\right)=v_{\mathrm{f}, m} \cdot \exp \left[-\frac{1}{a_{m}}\left(\frac{\rho_{m, i}(k)}{\rho_{\mathrm{cr}, m}}\right)^{a_{m}}\right]
$$

Note that the Metanet model (2)-(5) presented above is the basic model without geometry changes such as onramps, off-ramps, splits, or merges. However, the model can be extended to include those cases (see [5] for details). In the sequel, for the sake of simplicity but without loss of generality, we consider only one link, and therefore the index $m$ is dropped.

\section{TS FUZZY REPRESENTATION OF THE METANET MODEL}

In this section, a TS fuzzy model that exactly represents the Metanet model presented in the previous section is developed. Before the TS model is derived, the set of 
TABLE I

VARIABLES IN THE TRAFFIC MODEL.

\begin{tabular}{l|l|l} 
Symbol & Variable & Units \\
\hline$k$ & time step & - \\
$i$ & segment index & - \\
$\rho_{m, i}(k)$ & traffic density & $\mathrm{veh} / \mathrm{km} /$ lane \\
$v_{m, i}(k)$ & space-mean speed & $\mathrm{km} / \mathrm{h}$ \\
$q_{m, i}(k)$ & traffic volume or flow & $\mathrm{veh} / \mathrm{h}$ \\
\hline
\end{tabular}

TABLE II

PARAMETERS OF THE TRAFFIC MODEL.

\begin{tabular}{l|l|l|l} 
Symbol & Parameter & Value & Units \\
\hline$L_{m}$ & length of segment & 0.5 & $\mathrm{~km}$ \\
$\lambda_{m}$ & number of lanes & 3 & - \\
$v_{\mathrm{f}, m}$ & free flow speed & 102 & $\mathrm{~km} / \mathrm{h}$ \\
$\rho_{\mathrm{cr}, m}$ & critical density & 30 & $\mathrm{veh} / \mathrm{km} / \mathrm{lane}$ \\
$\tau$ & time constant & 18 & $\mathrm{~s}$ \\
$v$ & anticipation constant & 60 & $\mathrm{~km} / \mathrm{h}$ \\
$\kappa$ & constant & 40 & $\mathrm{veh} / \mathrm{km}$ \\
$a_{m}$ & parameter & 2.34 & - \\
$v_{\min }$ & minimum velocity & 7.4 & $\mathrm{~km} / \mathrm{h}$ \\
$v_{\max }$ & maximum velocity & 200 & $\mathrm{~km} / \mathrm{h}$ \\
$\rho_{\min }$ & minimum density & 0 & $\mathrm{veh} / \mathrm{km} / \mathrm{lane}$ \\
$\rho_{\max }$ & maximum density & 150 & $\mathrm{veh} / \mathrm{km} / \mathrm{lane}$ \\
$T$ & sampling time & 10 & $\mathrm{~s}$ \\
\hline
\end{tabular}

equations of the METANET model, i.e., equations (2), (3), (4), and (5), have to be written as a state space representation of a nonlinear system. Since in general TS fuzzy models do not use algebraic equations, $q_{i}(k)$ in (2) is eliminated, by substituting it into (3). The information needed from the neighboring segments, namely $\rho_{i+1}(k)$ and $v_{i-1}(k)$, is treated as input to the model. After some algebraic manipulations, the Metanet model can be expressed as a state equation with an affine term as follows

$$
\begin{aligned}
\left(\begin{array}{c}
\rho_{i}(k+1) \\
v_{i}(k+1)
\end{array}\right) & =\left(\begin{array}{cc}
1-\frac{T}{L} v_{i}(k) & 0 \\
\frac{v T}{\tau \cdot L} \frac{1}{\rho_{i}(k)+\kappa} & 1-\frac{T}{\tau}-\frac{T}{L} v_{i}(k)
\end{array}\right)\left(\begin{array}{l}
\rho_{i}(k) \\
v_{i}(k)
\end{array}\right) \\
& +\left(\begin{array}{cc}
\frac{T}{L} \rho_{i-1}(k) & 0 \\
\frac{T}{L} v_{i}(k) & -\frac{v T}{\tau \cdot L} \frac{1}{\rho_{i}(k)+\kappa}
\end{array}\right)\left(\begin{array}{l}
v_{i-1}(k) \\
\rho_{i+1}(k)
\end{array}\right) \\
& +\left(\begin{array}{c}
0 \\
\frac{T}{\tau} V\left(\rho_{i}(k)\right)
\end{array}\right) \\
V\left(\rho_{i}\right) & =v_{\mathrm{f}} \cdot \exp \left[-\frac{1}{a_{m}}\left(\frac{\rho_{i}}{\rho_{\mathrm{cr}}}\right)^{a_{m}}\right.
\end{aligned}
$$

The expression above exactly represents the METANET model. Now we can proceed with the development of the TS fuzzy representation of this model.

\section{A. TS fuzzy model construction}

Consider a nonlinear system described by the following state space $\operatorname{model}^{1}$ :

$$
\begin{aligned}
x(k+1) & =f(z) x(k)+g(z) u(k)+a(z) \\
y(k) & =h_{\text {out }}(z) x(k)
\end{aligned}
$$

Here, $f, g$, and $a$ are smooth nonlinear matrix and vector functions, respectively, $x \in \mathbb{R}^{n}$ is the state vector, $u \in \mathbb{R}^{m}$

\footnotetext{
${ }^{1}$ In the output equation we use $h_{\text {out }}$ to denote the nonlinear function and not $h$ as could be expected, since the symbol $h$ is used to denote membership functions.
}

the input vector, $y \in \mathbb{R}^{q}$ the measurement vector, and $z=$ $\left[\begin{array}{lll}z_{1}(k) & \cdots & z_{p}(k)\end{array}\right]^{\mathrm{T}}$ a given vector function ${ }^{2}$ of $x, y$, and $u$; $z$ is called the vector of scheduling variables. All variables $x$, $y, u$ are assumed to be bounded and to belong to a compact set $\mathbb{C}_{x y u}$.

There exist several approaches to obtain TS fuzzy models that exactly represent or approximate a given nonlinear system. A simple, yet effective approach is to use Taylor series expansion in several operating points, thereby obtaining local linear or affine models [16]. These models are then combined using fuzzy if-then rules to obtain an approximation of the nonlinear system. As the number of linearization points increases, so does the accuracy of the approximation. However, an increase in the number of local models implies a larger computational cost when designing observers. Another shortcoming of the approach is that there are no general guidelines on how to chose the linearization points.

A second approach to obtain a TS fuzzy approximation of a nonlinear model is to approximate the nonlinear matrixvalued functions $f, g$, and $h_{\text {out }}$ of (7) over intervals by aggregates of weighted constant matrices [17]. In [17] Gaussian membership functions have been used, and the number of operating points and the parameters of the Gaussians have been computed such that a specified approximation accuracy is achieved. However, this approach has the same shortcomings as the previous one: for an accurate approximation, a large number of local models is necessary.

The methods presented above can be used to construct TS models that are approximations of a given nonlinear system. An exact TS representation of a nonlinear system can be obtained using the sector nonlinearity approach [18]. The basic idea of the sector nonlinearity approach is to represent each of the non-constant terms in the matrix functions $f$, $g$, and $h_{\text {out }}$, and the vector function $a$ of the model as the convex combination of two constant terms and to build the set of fuzzy rules as all combinations of the so-obtained terms. Therefore, in this approach, the number of rules is determined by the number of non-constant terms in the matrix functions.

The multiplications of the state variables have the following effect: compared to the sector nonlinearity approach, a larger number of operating points is needed to obtain a TS model that accurately approximates the METANET model when the first two approaches discussed above are used. As already mentioned, using more operating points leads to a larger number of local models and a greater computational load when designing the observers. Therefore, we use the sector nonlinearity approach to construct the TS fuzzy representation of the METANET model. This goes as follows.

First consider the state equation of the nonlinear system (7). The sector nonlinearity approach requires the nonlinear

\footnotetext{
${ }^{2}$ Each element of the vector $z$ is time-dependent, i.e., $z$ should be denoted as $z(k)$. For the simplicity of notation, the explicit time-dependence is omitted in this paper.
} 
functions to be bounded. Therefore, we consider the nonconstant terms in either $f, g$, or $a$ of (7), and we represent them by $\mathrm{nl}_{j}(\cdot) \in\left[\underline{\mathrm{nl}}_{j}, \overline{\mathrm{nl}}_{j}\right], j=1,2, \ldots, p$ where $\underline{\mathrm{nl}}_{j}$ and $\overline{\mathrm{nl}}_{j}$ are respectively the lower and upper bound of the $j$-th term. Now, for each nonlinearity $\mathrm{nl}_{j}$, we construct two weighting functions as follows

$$
\begin{gathered}
w_{0}^{j}(\cdot)=\frac{\overline{\mathrm{nl}}_{j}-\mathrm{nl}_{j}(\cdot)}{\overline{\mathrm{nl}}_{j}-\underline{\mathrm{nl}}_{j}} \quad w_{1}^{j}(\cdot)=1-w_{0}^{j}(\cdot) \\
\quad \text { for } j=1,2, \ldots, p
\end{gathered}
$$

We can see that for each non-constant term, the two weighting functions $w_{0}^{j}$ and $w_{1}^{j}$ are normalized, i.e., $w_{0}^{j}\left(\mathrm{nl}_{j}(\cdot)\right)+$ $w_{1}^{j}\left(\mathrm{nl}_{j}(\cdot)\right)=1$, for any $\mathrm{nl}_{j}(\cdot)$. To define the membership functions we consider all possible products of the weight functions $w_{\ell}^{j}$ for $j \in\{1,2, \ldots, p\}$ and $\ell \in\{0,1\}$. This results in $2^{p}$ membership functions of the form

$$
h_{i}(z)=\prod_{j=1}^{p} w_{\ell}^{j}\left(z_{j}\right)
$$

for $i=1,2, \cdots, 2^{p}, \ell \in\{0,1\}$. These membership functions are normal, i.e., $h_{i}(z) \geq 0$ and $\sum_{i=1}^{r} h_{i}(z)=1, r=2^{p}$, where $r$ is the number of rules. Then the fuzzy representation of (7) is given as

$$
x(k+1)=\sum_{i=1}^{r} h_{i}(z)\left(A_{i} x(k)+B_{i} u(k)+a_{i}\right)
$$

where $A_{i}, B_{i}$, and $a_{i}, i=1,2, \ldots, r$ are matrices and vectors of proper dimensions, obtained by substituting the nonlinear terms $\mathrm{nl}_{j}(\cdot)$ by either $\underline{\mathrm{nl}}_{j}$ or $\overline{\mathrm{nl}}_{j}$ depending on whether $w_{0}^{j}$ or $w_{1}^{j}$ is selected in the membership function $h_{i}$ of rule $i$.

The TS representation of the output function can be obtained in a similar way.

\section{B. TS fuzzy representation of the METANET model}

To construct the TS fuzzy representation of (6) using the sector nonlinearity approach, it is necessary to assume that the values of the variables $\rho_{i-1}(k), \rho_{i}(k)$, and $v_{i}(k)$ are bounded, $\rho_{i-1}(k) \in\left[\rho_{i-1, \min }, \rho_{i-1, \max }\right], \rho_{i}(k) \in\left[\rho_{i, \min }, \rho_{i, \max }\right]$, and $v_{i}(k) \in\left[v_{i, \min }, v_{i, \max }\right]$, for all $k$. This assumption is reasonable since a freeway segment always has capacity limits. Furthermore, when the segment is in congestion, the space-mean speed will be very small, while there is always an upper limit of the speed of a car on a freeway.

First, we consider the state equation of (6). There are four non-constant terms in the matrix functions $f, g$, and $a$, based on which the weighting functions are defined as follows

1) For the term $1-\frac{T}{L} v_{i}(k)$, the space-mean speed $v_{i}(k)$ has a maximum and minimum value of $v_{i, \max }$ and $v_{i, \min }$ respectively. Applying (8), one obtains $w_{0}^{1}\left(v_{i}(k)\right)=\frac{v_{i, \max }-v_{i}(k)}{v_{i, \max }-v_{i, \min }}$ and $w_{1}^{1}\left(v_{i}(k)\right)=1-w_{0}^{1}\left(v_{i}(k)\right)$; note that the nonlinearities $1-\frac{T}{L} v_{i}(k)$ and $1-\frac{T}{\tau}-\frac{T}{L} v_{i}(k)$ lead to the same weighting functions;

2) Similarly to the above, $\frac{1}{\rho_{i}(k)+\kappa}$ leads to

$$
\begin{aligned}
& w_{0}^{2}\left(\rho_{i}(k)\right)=\frac{\rho_{i}(k)-\rho_{i, \min }}{\rho_{i}(k)+\kappa} \frac{\rho_{i, \max }+\kappa}{\rho_{i, \max }-\rho_{i, \min }}, \\
& w_{1}^{2}\left(\rho_{i}(k)\right)=1-w_{0}^{2}\left(\rho_{i}(k)\right) ;
\end{aligned}
$$

3) The term $\exp \left[-\frac{1}{a_{m}}\left(\frac{\rho_{i}(k)}{\rho_{\text {cr }}}\right)^{a_{m}}\right]$ appearing in $V\left(\rho_{i}\right)$ is expressed using the weighting functions

$$
\begin{aligned}
& w_{0}^{3}\left(\rho_{i}(k)\right)=\frac{\exp \left[-\frac{1}{a_{m}}\left(\frac{\rho_{i, \min }}{\rho_{\mathrm{cr}}}\right)^{a_{m}}\right]-\exp \left[-\frac{1}{a_{m}}\left(\frac{\rho_{i}(k)}{\rho_{\mathrm{cr}}}\right)^{a_{m}}\right]}{\exp \left[-\frac{1}{a_{m}}\left(\frac{\rho_{i, \min }}{\rho_{\mathrm{cr}}}\right)^{a_{m}}\right]-\exp \left[-\frac{1}{a_{m}}\left(\frac{\rho_{i, \max }}{\rho_{\mathrm{cr}}}\right)^{a_{m}}\right]}, \\
& w_{1}^{3}\left(\rho_{i}(k)\right)=1-w_{0}^{3}\left(\rho_{i}(k)\right)
\end{aligned}
$$

4) The term $\rho_{i-1}(k)$ leads to the weighting functions

$$
\begin{aligned}
& w_{0}^{4}\left(\rho_{i-1}(k)\right)=\frac{\rho_{i-1, \max }-\rho_{i-1}(k)}{\rho_{i-1, \max }-\rho_{i-1, \min },} \\
& w_{1}^{5}\left(\rho_{i-1}(k)\right)=1-w_{0}^{5}\left(\rho_{i-1}(k)\right) .
\end{aligned}
$$

Based on the above four non-constant terms, to describe all possible combinations, a fuzzy system with $2^{4}=16$ rules is necessary.

Consider now the output equation

$$
\begin{aligned}
y(k) & =\left(\begin{array}{c}
q_{i}(k) \\
v_{i}(k)
\end{array}\right) \\
& =\left(\begin{array}{cc}
v_{i}(k) \lambda & 0 \\
0 & 1
\end{array}\right)\left(\begin{array}{l}
\rho_{i}(k) \\
v_{i}(k)
\end{array}\right) .
\end{aligned}
$$

The measurement matrix has one non-constant term, namely $v_{i}(k)$. But since $v_{i}(k)$ in the measurement matrix is the same as that of the system equation, the same weighting function as above can be used. Moreover, the speed $v_{i}(k)$ is also assumed to be measured, which means the membership functions of the measurement do not depend on states that have to be estimated.

Using the weighting functions developed above, the consequent models of the fuzzy rules can be written as

$$
\begin{aligned}
x(k+1) & =A_{i} x(k)+B_{i} u(k)+a_{i} \\
y(k) & =C_{i} x(k)
\end{aligned}
$$

where

$$
x(k)=\left(\begin{array}{c}
\rho_{i}(k) \\
v_{i}(k)
\end{array}\right) \quad u(k)=\left(\begin{array}{c}
v_{i-1}(k) \\
\rho_{i+1}(k)
\end{array}\right)
$$

and $A_{i}, B_{i}, a_{i}$, and $C_{i}$ are obtained by substituting the minimum or maximum values corresponding to the weighting functions used in rule $i$ into the functions $f, g, h_{\text {out }}$, and $a$.

The TS fuzzy model of the Metanet is then expressed as

$$
\begin{aligned}
x(k+1) & =\sum_{i=1}^{r} h_{i}(z)\left(A_{i} x(k)+B_{i} u(k)+a_{i}(k)\right) \\
y(k) & =\sum_{i=1}^{r} h_{i}(z) C_{i} x(k)
\end{aligned}
$$

This concludes the TS fuzzy representation of the METANET model.

\section{ObServer Design For the TS Metanet MOdel}

In general, an observer designed for the model (13) has the form

$$
\begin{gathered}
\hat{x}(k+1)=\sum_{i=1}^{r} h_{i}(\hat{z})\left[A_{i} \hat{x}(k)+B_{i} u(k)+a_{i}\right. \\
\left.\quad+K_{i}(y(k)-\hat{y}(k))\right] \\
\hat{y}(k)=\sum_{i=1}^{r} h_{i}(\hat{z}) C_{i} \hat{x}
\end{gathered}
$$


where $\hat{z}$ denotes the estimated scheduling vector and $K_{i}, i=$ $1, \ldots, r$, are the observer gains. The observer design problem is to calculate the values of $K_{i}, i=1, \ldots, r$ such that the estimation error converges to zero. The estimation error can be written as

$$
e(k)=\hat{x}(k)-x(k)
$$

Substituting (13) and (14) into (15) yields

$$
\begin{aligned}
& e(k)=\sum_{i=1}^{r} h_{i}(z)\left[A_{i} x(k)+B_{i} u(k)+a_{i}\right] \\
& -\sum_{i=1}^{r} h_{i}(\hat{z})\left[A_{i} \hat{x}(k)+B_{i} u(k)+a_{i}+K_{i}(y(k)-\hat{y}(k))\right]
\end{aligned}
$$

Adding to and subtracting from the right-hand side of the above equation $\sum_{i=1}^{r} h_{i}(\hat{z})\left(A_{i} x(k)+B_{i} u(k)+a_{i}\right)$, after some algebraic manipulations we obtain

$$
\begin{gathered}
e(k+1)=\sum_{i=1}^{r} h_{i}(\hat{z})\left[A_{i} e(k)-K_{i}(y(k)-\hat{y}(k))\right] \\
+\sum_{i=1}^{r}\left(h_{i}(z)-h_{i}(\hat{z})\right)\left[A_{i} x(k)+B_{i} u(k)+a_{i}(k)\right]
\end{gathered}
$$

Since the speed is measured, the membership functions of the measurement model do not depend on the estimated states. Therefore, we can rewrite (16) as

$$
\begin{aligned}
& e(k+1)=\sum_{i=1}^{r} \sum_{j=1}^{r} h_{i}(\hat{z}) h_{j}(z)\left[A_{i}-K_{i} C_{j}\right] e(k) \\
& +\sum_{i=1}^{r}\left(h_{i}(z)-h_{i}(\hat{z})\right)\left[A_{i} x(k)+B_{i} u(k)+a_{i}(k)\right]
\end{aligned}
$$

In order for the estimation error to converge to zero, the observer gains $K_{i}$ have to be calculated such that the first term of (17) converges to zero and such that the disturbance due to the second term, $h_{i}(z)-h_{i}(\hat{z})$ becomes zero as $\hat{z}$ approaches $z$.

The observer gains $K_{i}$ are usually computed using stability conditions developed for TS systems. The estimation error dynamics (17) is asymptotically stable, i.e., the estimation error converges to zero if there exists a positive definite matrix $P$ such that [13]

$$
\begin{array}{r}
G_{i i}^{T} P G_{i i}-P<0 \\
\frac{\left(G_{i j}+G_{j i}\right)^{T}}{2} P \frac{\left(G_{i j}+G_{j i}\right)}{2}-P<0
\end{array}
$$

for all $i, j$ such that $i<j$ and $\exists z$ s.t. $h_{i}(z) h_{j}(z) \neq 0$

where $G_{i j}=A_{i}-K_{i} C_{j}$. The inequalities above can be transformed into the following LMI problem:

Find a positive definite matrix $P$ and matrices $M_{i}$, where $M_{i}=P K_{i}, i=1, \ldots, r$, such that

$$
\begin{aligned}
& \left(\begin{array}{cc}
P & 2 L_{i i}^{T} \\
2 L_{i i} & P
\end{array}\right)>0 \\
& \left(\begin{array}{cc}
P & \left(L_{i j}+L_{j i}\right)^{T} \\
\left(L_{i j}+L_{j i}\right) & P
\end{array}\right)>0
\end{aligned}
$$

for all $i, j$ such that $i<j$ and $\exists z$ s.t. $h_{i}(z) h_{j}(z) \neq 0$ where $L_{i j}=\left(P A_{i}-M_{i} C_{j}\right) / 2$.
The condition (19) above ensures the asymptotic stability of the first term of the right-hand side of (17). The asymptotic stability of (17) can be guaranteed using stability conditions for uncertain fuzzy systems (see [19]). Since (19) are robust stability conditions and provided the initial estimate is close enough to the true state, (17) is stable [20].

The LMIs above can e.g. be solved using the Sedumi solver of YALMIP [21]. Next the values of $K_{i}$ are substituted into the observer model.

The approach presented above can easily be extended to include the node equations of the METANET model, which implies that the proposed approach is not only also applicable to freeway stretches but also to (complex) freeway networks.

Note that the fuzzy model is also observable if only the flow is measured, (i.e., the speed is not measured), as long as neither the flow nor the speed on a segment is zero. Moreover, under these conditions it is possible to design a fuzzy observer similar to (14) that can estimate both the speed and the density. This indicates that it is possible to design observers in a distributed fashion for a whole stretch or even a whole network, given that the neighboring observers communicate the estimated states among them.

\section{Simple CASE STUDY}

Now the proposed approach is illustrated for simple case study in which we consider one particular segment $i$. The true initial state of the segment is $x=\left[\begin{array}{ll}10 & 20\end{array}\right]^{\mathrm{T}}$. The boundary inputs for the segment were constructed such that the downstream speed was equal to the initial speed of the segment plus a random uniform noise signal with an amplitude of $15 \mathrm{~km} / \mathrm{h}$, and such that the upstream density was equal to the initial density of the segment plus a random uniform noise signal with an amplitude of $15 \mathrm{veh} / \mathrm{km} / \mathrm{lane}$. The observer has been simulated using the initial estimate $x=\left[\begin{array}{ll}20 & 100\end{array}\right]^{\mathrm{T}}$. The output of the TS fuzzy representation of the Metanet model is shown in Figure 1. The estimation error using the observer is shown in Figure 2. As expected, the estimation error converges to zero.

The simulation and estimation reported here have been performed on a PC with an Intel T9300 $2.5 \mathrm{GHz}$ processor and 3GB RAM. The total computation time, including the computation of the observer gains $(1.75 \mathrm{~s}$, done offline, before the actual estimation), simulation of the model and estimation of the states was $2.26 \mathrm{~s}$. To compute the estimate in one time step on average requires $0.0042 \mathrm{~s}$, with $0.008 \mathrm{~s}$ being the maximum time that was encountered. These values are well below the typical sampling times for freeway traffic networks (which currently are typically in the range of several tens of seconds to minutes). This clearly indicates that the proposed observer is applicable online.

\section{CONCLUSIONS}

A discrete-time Takagi-Sugeno (TS) fuzzy observer has been proposed in this paper for the METANET traffic model. An exact TS representation of the METANET model has been obtained using the sector nonlinearity approach. The observer has been designed based on the TS fuzzy representation of 

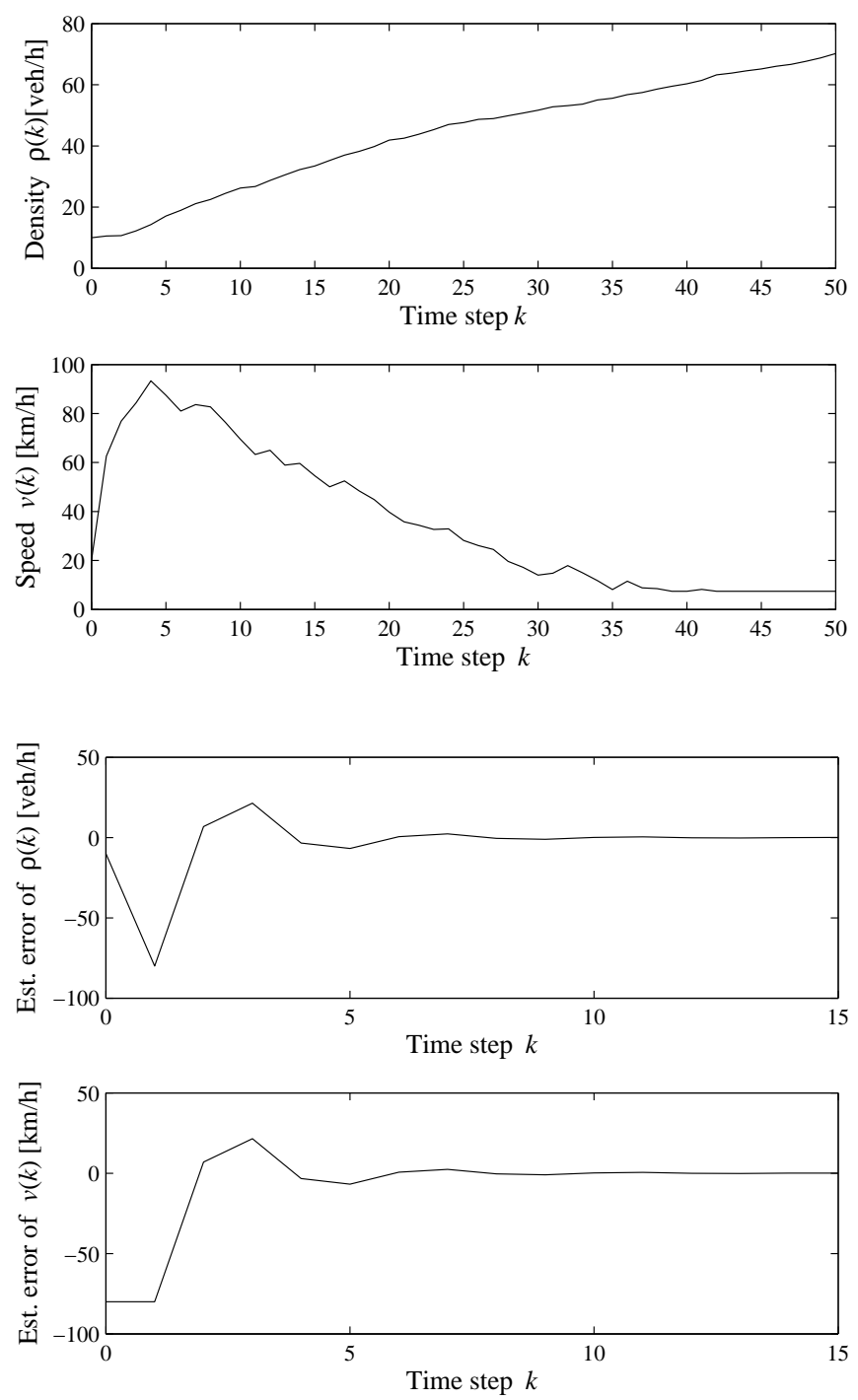

Fig. 2. Estimation error using the TS fuzzy observer.

the Metanet model for one segment of highway stretch. The designed observer is able to estimate the non-measurable traffic states.

In our future research, we will investigate how the performance of proposed observer compares to that of other types of observers that can be applied to the METANET model such as extended Kalman filters, unscented Kalman filters, or particle filters (see also [6]-[10]), in particular for models of real-life networks and using real measurement data as input. We will also consider robust TS fuzzy observer design in order to handle uncertainties in the METANET model, as well as TS fuzzy observers for other traffic flow models.

\section{ACKNOWLEDGMENT}

The first author gratefully acknowledges the support of the Government of the Republic of Indonesia, Ministry of Communication and Information Technology.

\section{REFERENCES}

[1] S. Hoogendoorn and P. Bovy, "State-of-the-art of vehicular traffic flow modelling," Proceedings of the Institution of Mechanical Engineers,
Part I: Journal of Systems and Control Engineering, vol. 215, no. 4, pp. 283-303, Jan. 2001.

[2] A. Messmer and M. Papageorgiou, "Automatic control methods applied to freeway network traffic," Automatica, vol. 30, no. 4, pp. 691702, 1994.

[3] M. van den Berg, A. Hegyi, B. De Schutter, and J. Hellendoorn, "Integrated traffic control for mixed urban and freeway networks: A model predictive control approach," European Journal of Transport and Infrastructure Research, vol. 7, no. 3, pp. 223-250, Sept. 2007.

[4] A. Kotsialos and M. Papageorgiou, "Motorway network traffic control systems," European Journal of Operational Research, vol. 152, no. 2, pp. 321-333, 2004, new Technologies in Transportation Systems.

[5] M. Papageorgiou, J.-M. Blosseville, and H. Hadj-Salem, "Macroscopic modelling of traffic flow on the Boulevard Périphérique in Paris," Transportation Research Part B: Methodological, vol. 23, no. 1, pp. 29-47, February 1989.

[6] Y. Wang and M. Papageorgiou, "Real-time freeway traffic state estimation based on extended Kalman filter: A general approach," Transportation Research Part B: Methodological, vol. 39, no. 2, pp. 141-167, 2005.

[7] L. Mihaylova, R. Boel, and A. Hegyi, "An unscented Kalman filter for freeway traffic estimation," in Proceedings of the IFAC Symposium on Control in Transportation Systems, Delft, The Netherlands, Aug. 29-31, 2006 2006, pp. 31-36.

[8] — - "Freeway traffic estimation within particle filtering framework," Automatica, vol. 43, no. 2, pp. 290-300, 2007.

[9] Y. Wang, M. Papageorgiou, A. Messmer, P. Coppola, A. Tzimitsi, and A. Nuzzolohegyi, "An adaptive freeway traffic state estimator," Automatica, vol. 45, no. 1, pp. 10-24, 2009.

[10] A. Hegyi, D. Girimonte, R. Babuška, and B. De Schutter, "A comparison of filter configurations for freeway traffic state estimation," in Proceedings of IEEE Intelligent Transportation Systems Conference (ITSC '06), Toronto, Canada, Sept. 2006, pp. 1029-1034.

[11] T. Takagi and M. Sugeno, "Fuzzy identification of systems and its applications to modeling and control," IEEE Transactions on Systems, Man, and Cybernetics, vol. 15, no. 1, pp. 116-132, 1985.

[12] P. Bergsten, R. Palm, and D. Driankov, "Observers for Takagi-Sugeno fuzzy systems," IEEE Transactions on Systems, Man, and Cybernetics, Part B: Cybernetics, vol. 32, no. 1, pp. 114-121, feb 2002.

[13] K. Tanaka, T. Ikeda, and H. O. Wang, "Fuzzy regulators and fuzzy observers: relaxed stability conditions and LMI-based designs," IEEE Transactions on Fuzzy Systems, vol. 6, no. 2, pp. 250-265, May 1998.

[14] Zs. Lendek, R. Babuška, and B. De Schutter, "Fuzzy models and observers for freeway traffic state tracking," in Proceedings of the 2010 American Control Conference, Baltimore, Maryland, 2010, pp. 2278-2283.

[15] A. Kotsialos, M. Papageorgiou, C. Diakaki, Y. Pavlis, and F. Middelham, "Traffic flow modeling of large-scale motorway networks using the macroscopic modeling tool METANET," IEEE Transactions on Intelligent Transportation Systems, vol. 3, no. 4, pp. 282-292, Dec 2002.

[16] H. Wang, J. Li, D. Niemann, and K. Tanaka, "T-S fuzzy model with linear rule consequence and PDC controller: a universal framework for nonlinear control systems," in Proceedings of the Ninth IEEE International Conference on Fuzzy Systems (FUZZ IEEE 2000), San Antonio, TX, USA, May 2000, pp. $549-554$.

[17] K. Kiriakidis, "Nonlinear modeling by interpolation between linear dynamics and its application in control," Journal of Dynamic Systems, Measurement, and Control, vol. 129, no. 6, pp. 813-824, 2007.

[18] H. Ohtake, K. Tanaka, and H. O. Wang, "Fuzzy modeling via sector nonlinearity concept," Integrated Computer-Aided Engineering, vol. 10, no. 4, pp. 333-341, Jan. 2003.

[19] D. Ichalal, B. Marx, J. Ragot, and D. Maquin, "State and unknown input estimation for nonlinear systems described by Takagi-Sugeno models with unmeasurable premise variables," in Proceedings of the 17th Mediterranean Conference on Control and Automation, Thessaloniki, Greece, June 2009, pp. 217-222.

[20] P. Bergsten, "Observers and controllers for Takagi-Sugeno fuzzy systems," PhD Thesis, Örebro University, Sweden, September 2001.

[21] J. Löfberg, "YALMIP: A toolbox for modeling and optimization in MATLAB," in Proceedings of the International Symposium on Computer Aided Control Systems Design, Taipei, Taiwan, 2004, pp. 284-289. 